\title{
HIGHER ORDER CORRECTIONS IN THE CoLoRFulNNLO FRAMEWORK*
}

\author{
Gábor Somogyi, Adam Kardos, Zoltán SzÖr \\ ZOLTÁN TRÓCSÁNYI \\ University of Debrecen and MTA-DE Particle Physics Research Group \\ P.O. Box 105, 4010 Debrecen, Hungary \\ (Received March 31, 2017)
}

We discuss the CoLorFulNNLO method for computing higher order radiative corrections to jet cross sections in perturbative QCD. We apply our method to the calculation of event shapes and jet rates in three-jet production in electron-positron annihilation. We validate our code by comparing our predictions to previous results in the literature and present the jet cone energy fraction distribution at NNLO accuracy. We also present preliminary NNLO results for the three-jet rate using the Durham jet clustering algorithm matched to resummed predictions at NLL accuracy, and a comparison to LEP data.

DOI:10.5506/APhysPolB.48.????

\section{Introduction}

The strong coupling $\alpha_{\mathrm{S}}$ is one of the fundamental parameters of the Standard Model of particle physics thus, its precise determination is mandatory. Nowadays, event shapes and jet rates measured in three-jet formation in electron-positron annihilation are still among the most precise tools used for accurate extractions of $\alpha_{\mathrm{s}}$ from data. In these analyses, the measurement of $\alpha_{\mathrm{s}}$ involves fitting theoretical predictions for a given observable and collider energy to observations. Hence, theoretical input is essential and the goodness of the fitting procedure relies heavily on the quality of the theoretical predictions used.

In high-energy particle physics, we may approach the quality of theoretical predictions from two perspectives. First, the general framework for performing calculations is perturbation theory, where in the case of QCD the

* Presented by G. Somogyi at the Cracow Epiphany Conference "Particle Theory Meets the First Data from LHC Run 2", Kraków, Poland, January 9-12, 2017. 
perturbative parameter is the strong coupling, $\alpha_{\mathrm{s}}$. Due to the complexity of calculations, only the first few terms of the perturbative series can be evaluated and this truncation introduces a theoretical uncertainty manifested by the dependence of the predictions on non-physical parameters such as the renormalization and factorization scales. Thus, one way of increasing the theoretical precision of the calculations is by including exact higher order corrections in perturbation theory. Second, the actual calculations of physical observables involve numerical integrations over the physical phase space and this introduces a statistical uncertainty in the predictions. Hence, formal higher order precision must be supplemented by a good numerical accuracy to obtain results that are useful for experimental needs.

For three-jet production in electron-positron annihilation, all matrix elements necessary for the computation of next-to-next-to-leading order (NNLO) corrections have been known in the literature for some time [1-4] and indeed NNLO corrections to several event shapes [5-8] and jet rates have been evaluated $[9,10]$. Hence, this process is not only interesting from a phenomenological point of view, but it also provides an ideal testing ground for new computational methods at this order in perturbation theory. In this contribution, we summarize a completely local subtraction method, dubbed CoLorFulNNLO, for computing QCD corrections to jet cross sections at NNLO accuracy and present the application of our framework to threejet production in electron-positron annihilation. Our method demonstrates excellent numerical stability and accuracy for the considered observables.

\section{The CoLorFulNNLO method}

In perturbative QCD the expansion of a jet cross section defined by some physical quantity $J$ can be formally written up to NNLO accuracy as

$$
\sigma[J]=\sigma^{\mathrm{LO}}[J]+\sigma^{\mathrm{NLO}}[J]+\sigma^{\mathrm{NNLO}}[J]+\ldots
$$

Focusing on the production of $m$ jets from a colorless initial state, the leading order (LO) cross section is simply given by integrating the fully differential Born cross section for the production of $m$ partons over the $m$-parton phase space defined by the observable $J$

$$
\sigma^{\mathrm{LO}}[J]=\int_{m} \mathrm{~d} \sigma_{m}^{\mathrm{B}} J_{m} .
$$

The next-to-leading order (NLO) correction can be written as the sum of two terms

$$
\sigma^{\mathrm{NLO}}[J]=\int_{m+1} \mathrm{~d} \sigma_{m+1}^{\mathrm{R}} J_{m+1}+\int_{m} \mathrm{~d} \sigma_{m}^{\mathrm{V}} J_{m},
$$


and is finite for any infrared-safe observable by the general theorem of Kinoshita, Lee and Nauenberg (KLN). Although the KLN theorem guarantees the finiteness for the sum of the real emission $\left(\sigma_{m+1}^{\mathrm{R}}\right)$ and virtual $\left(\sigma_{m}^{\mathrm{V}}\right)$ corrections, it does not say anything about the contributions separately which are indeed infinite in four spacetime dimensions. Using conventional dimensional regularization in $d=4-2 \epsilon$ dimensions to regularize the two pieces, the singularities become poles in $\epsilon$, which nevertheless cancel between the two contributions in the final result. However, this cancellation is not manifest. On the one hand, the singularities in the real emission part have a kinematic origin: they are due to divergent phase-space integrals when one final state parton becomes unresolved. On the other hand, the $\epsilon$-poles in the virtual correction arise from the integration over the loop momentum. In general, the squared matrix elements and observables in QCD are much too complicated to perform an analytic calculation in $d$ dimensions, and our aim is to carry out the computations in four dimensions using the Monte Carlo techniques. To do so, the real and virtual emission contributions have to be made finite separately which we achieve by local subtractions. In this method, an approximate differential cross section, $\mathrm{d} \sigma_{m+1}^{\mathrm{R}, \mathrm{A}_{1}}$, is subtracted from the real emission contribution. This approximate cross section is constructed carefully to have the same kinematic singularity structure (in $d$ dimensions) as the real emission cross section. Thus, the difference is free of non-integrable kinematic singularities and the phase-space integral can be evaluated in four dimensions using the standard Monte Carlo techniques. The poles appearing in the virtual contribution are then removed by adding back the approximate cross section after integrating over the momentum and summing over the quantum numbers (color, flavor) of the unresolved particle (these operations are collectively denoted by $\int_{1}$ ). Then the NLO correction takes the form of

$$
\begin{aligned}
\sigma^{\mathrm{NLO}}[J]= & \int_{m+1}\left[\mathrm{~d} \sigma_{m+1}^{\mathrm{R}} J_{m+1}-\mathrm{d} \sigma_{m+1}^{\mathrm{R}, \mathrm{A}_{1}} J_{m}\right]_{d=4} \\
& +\int_{m}\left[\mathrm{~d} \sigma_{m}^{\mathrm{V}} J_{m}+\int_{1} \mathrm{~d} \sigma_{m+1}^{\mathrm{R}, \mathrm{A}_{1}} J_{m}\right]_{d=4},
\end{aligned}
$$

where now both contributions are finite as discussed. Several explicit constructions are available for the approximate cross section $\mathrm{d} \sigma_{m+1}^{\mathrm{R}, \mathrm{A}_{1}}$ in the literature [11-15].

The NNLO correction is composed of three different contributions

$$
\sigma^{\mathrm{NNLO}}[J]=\int_{m+2} \mathrm{~d} \sigma_{m+2}^{\mathrm{RR}} J_{m+2}+\int_{m+1} \mathrm{~d} \sigma_{m+1}^{\mathrm{RV}} J_{m+1}+\int_{m} \mathrm{~d} \sigma_{m}^{\mathrm{VV}} J_{m} .
$$


The first term is the double real (RR) piece which involves tree-level squared matrix elements with $m+2$-parton kinematics and develops singularities when one or two partons become unresolved. The second term is the realvirtual one (RV) and contains the interference of one-loop and tree-level matrix elements with $m+1$-parton kinematics. This contribution develops both kinematic singularities when a parton becomes unresolved and also contains explicit $\epsilon$-poles coming from one-loop amplitudes. Finally, the third term is the double virtual (VV) contribution, which includes the interference of the $m$-parton two-loop and tree-level matrix elements as well as the square of the $m$-parton one-loop matrix element. This contribution is free from kinematic singularities (the infrared-safe jet function screens any remaining divergences of the squared matrix elements), but it contains explicit $\epsilon$-poles which come from integrations over loop momenta.

The idea behind the CoLorFulNNLO method is to define completely local subtraction terms for the NNLO correction in the same spirit as was done at NLO accuracy. Thus, the $m+2$-parton contribution is made finite by introducing local subtraction terms whose kinematic singularities exactly reproduce those of the double real emission matrix elements (in $d$ dimensions) in each single and double unresolved limit

$$
\begin{aligned}
\sigma_{m+2}^{\mathrm{NNLO}}[J]= & \int_{m+2}\left\{\mathrm{~d} \sigma_{m+2}^{\mathrm{RR}} J_{m+2}-\mathrm{d} \sigma_{m+2}^{\mathrm{RR}, \mathrm{A}_{2}} J_{m}\right. \\
& \left.-\left[\mathrm{d} \sigma_{m+2}^{\mathrm{RR}, \mathrm{A}_{1}} J_{m+1}-\mathrm{d} \sigma_{m+2}^{\mathrm{RR}, \mathrm{A}_{12}} J_{m}\right]\right\}_{d=4} .
\end{aligned}
$$

In Eq. (6), $\mathrm{d} \sigma_{m+2}^{\mathrm{RR}, \mathrm{A}_{2}}$ regularizes those singularities of the $\mathrm{RR}$ contribution which emerge in double unresolved limits, while $\mathrm{d} \sigma_{m+2}^{\mathrm{RR}, \mathrm{A}_{1}}$ serves as a local counterterm for single unresolved singularities. The last term, $\mathrm{d} \sigma_{m+2}^{\mathrm{RR}, \mathrm{A}_{12}}$, is introduced to remove both the kinematic singularities that develop in $\mathrm{d} \sigma_{m+2}^{\mathrm{RR}, \mathrm{A}_{2}}$ in single unresolved regions and also the singularities of $\mathrm{d} \sigma_{m+2}^{\mathrm{RR}, \mathrm{A}_{1}}$ in double unresolved ones. The precise definitions of all subtraction terms that appear in Eq. (6) were given in [16].

The $m+1$-parton contribution takes the form of

$$
\begin{aligned}
\sigma_{m+1}^{\mathrm{NNLO}}= & \int_{m+1}\left\{\left(\mathrm{~d} \sigma_{m+1}^{\mathrm{RV}}+\int_{1} \mathrm{~d} \sigma_{m+2}^{\mathrm{RR}, \mathrm{A}_{1}}\right) J_{m+1}\right. \\
& \left.-\left[\mathrm{d} \sigma_{m+1}^{\mathrm{RV}, \mathrm{A}_{1}}+\left(\int_{1} \mathrm{~d} \sigma_{m+2}^{\mathrm{RR}, \mathrm{A}_{1}}\right)^{\mathrm{A}_{1}}\right] J_{m}\right\}_{d=4},
\end{aligned}
$$


where the first line of Eq. (7) contains the RV contribution as well as the integrated form of the single unresolved subtraction term in Eq. (6), $\int_{1} \mathrm{~d} \sigma_{m+2}^{\mathrm{RR}, \mathrm{A}_{1}}$. The sum of these two terms is free of $\epsilon$-poles [14], however both terms still contain kinematical singularities when a parton becomes unresolved. These singularities are regularized by the local subtraction terms on the second line of Eq. (7). The exact definitions of these subtraction terms were presented in [17].

The last contribution to the NNLO correction is the $m$-parton one which contains the VV contribution along with the integrated forms of all remaining subtraction terms which we have not yet added back. Schematically, this can be written as

$$
\begin{aligned}
\sigma_{m}^{\mathrm{NNLO}}= & \int_{m}\left\{\mathrm{~d} \sigma_{m}^{\mathrm{VV}}+\int_{2}\left[\mathrm{~d} \sigma_{m+2}^{\mathrm{RR}, \mathrm{A}_{2}}-\sigma_{m+2}^{\mathrm{RR}, \mathrm{A}_{12}}\right]\right. \\
& \left.+\int_{1}\left[\mathrm{~d} \sigma_{m+1}^{\mathrm{RV}, \mathrm{A}_{1}}+\left(\int_{1} \mathrm{~d} \sigma_{m+2}^{\mathrm{RR}, \mathrm{A}_{1}}\right)^{\mathrm{A}_{1}}\right]\right\}_{d=4} J_{m} .
\end{aligned}
$$

Since the $m+2$ - and $m+1$-parton contributions in Eqs. (6) and (7) are both finite by construction, the finiteness of the $m$-parton piece in Eq. (8) is automatic and guaranteed by the KLN theorem. The various integrated approximate cross sections that appear in Eq. (8) were computed in a line of papers [18], culminating in the explicit demonstration of the finiteness of this contribution for electron-positron annihilation into three jets in [8]. Since the subtractions render all three contributions finite, they can be separately integrated numerically using standard Monte Carlo techniques. We stress that since all subtractions are completely local, the integrations may be performed with any convenient numerical procedure.

\section{Electron-positron annihilation into three jets}

We have implemented the CoLorFulNNLO scheme as outlined above into the fortran90 program library MCCSM (Monte Carlo for the CoLorFulNNLO Subtraction Method). The implementation is completely general for processes with colorless initial states, with only the squared matrix elements for a given process (including the color- and spin-correlated ones) as necessary inputs.

As a first application, we used our method and code to compute NNLO QCD corrections to physical observables in three-jet production in electronpositron annihilation $[7,8]$. Since these corrections are known for several quantities in the literature $[5,6,9,10]$ (see also [19] which describes the EERAD3 program implementing the computations of $[5,9]$ ), this provides 
an excellent opportunity to validate our method and the framework implementing it. Hence, we compared our predictions for the six standard event shape variables of thrust $(T), C$-parameter, total- and wide-jet broadening, heavy-jet mass and the two-to-three jet transition variable $y_{23}$ in the Durham jet clustering algorithm to the predictions of $[5,6]$. We performed the comparisons at the LEP2 energy of $\sqrt{s}=m_{Z}=91.2 \mathrm{GeV}$. The perturbative coefficients were defined using the normalization common at lepton-lepton colliders

$$
\frac{O}{\sigma_{0}} \frac{\mathrm{d} \sigma}{\mathrm{d} O}=\frac{\alpha_{\mathrm{s}}}{2 \pi} O A(O)+\left(\frac{\alpha_{\mathrm{s}}}{2 \pi}\right)^{2} O B(O)+\left(\frac{\alpha_{\mathrm{s}}}{2 \pi}\right)^{3} O C(O)+\mathcal{O}\left(\alpha_{\mathrm{s}}^{4}\right),
$$

where $\sigma_{0}$ is the leading order cross section for $e^{+} e^{-} \rightarrow$ hadrons and $O$ is the event shape variable for which we obtain the NNLO accurate prediction.

We present the comparison of our predictions to those of EERAD3 (denoted by $\mathrm{GGGH}^{1}$ ) and Ref. [6] (denoted $\mathrm{SW}^{2}$ ) for two representative cases, thrust $(\tau=1-T)$ and the $C$-parameter in Fig. 1 and Fig. 2. In these plots, the left-hand panels show the physical predictions for the observables at LO, NLO and NNLO accuracy, together with the data measured by the
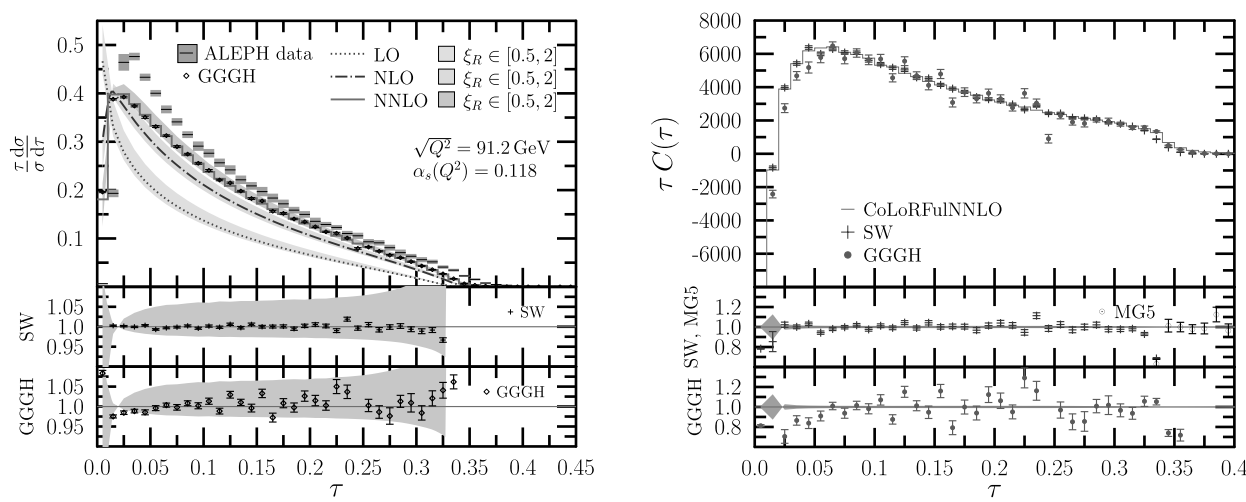

Fig. 1. Left: physical predictions for thrust $(\tau=1-T)$ at LO, NLO and NNLO accuracy in QCD with bands representing scale uncertainty. Data measured by the ALEPH Collaboration [20] is also shown. Right: the $\tau C(\tau)$ NNLO coefficient of the thrust distribution. In both figures, the lower panels show the ratio of the predictions of Ref. [6] (SW) and EERAD3 (GGGH) to CoLorFulNNLO. In the middle panel of the right figure, results from MadGraph5_aMC@NLO [23] (MG5) are also shown above the Born kinematic limit of $\tau>1 / 3$.

${ }^{1}$ We are grateful to G. Heinrich for providing the predictions of EERAD3 for us.

${ }^{2}$ In these comparisons, we use updated (with respect to those published in Ref. [6]) but unpublished predictions provided to us by S. Weinzierl. We are grateful to S. Weinzierl for providing these updated results for us. 
ALEPH Collaboration. The bands correspond to scale variations in the range of $\left[m_{Z} / 2,2 m_{Z}\right]$ with $\mu_{0}=m_{Z}$ chosen as the central scale. While these plots clearly show the convergence of the perturbative series for both the $\tau$ and $C$-parameter distributions as we go from LO to NLO and NNLO accuracy, the comparison with data also makes it evident that parton shower and nonperturbative corrections are sizable. In the lower panels, we plot the ratios of the predictions of GGGH (bottom panel) and SW (middle panel) normalized to our results and find agreement between the various computations, except at the kinematic limits of the distributions.
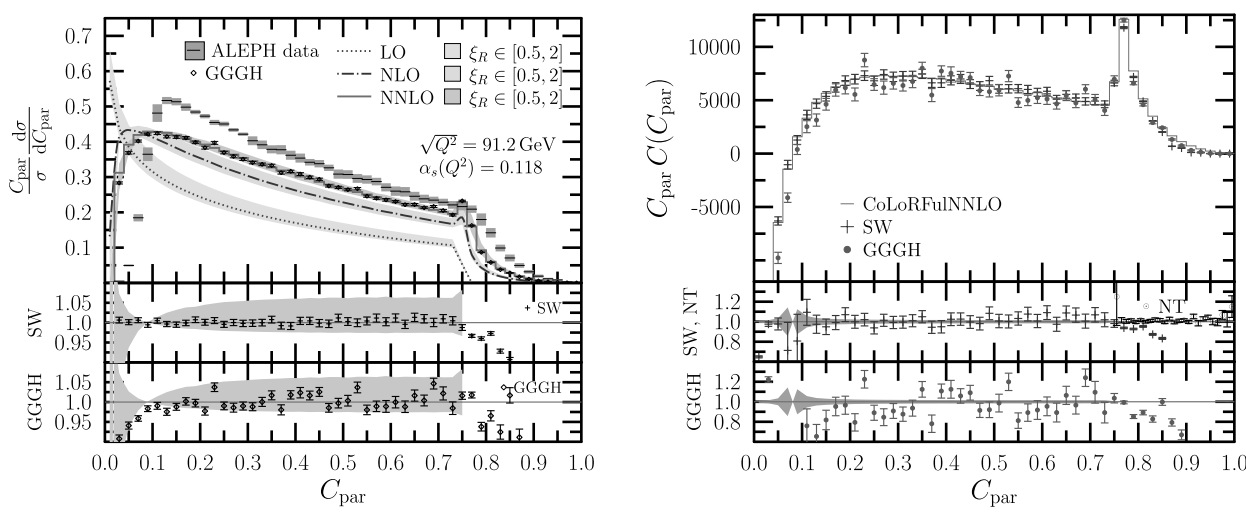

Fig. 2. The same as Fig. 1 for the $C$-parameter distribution. In the middle panel of the right figure, results from Ref. [22] (NT) are also shown above the Born kinematic limit of $C_{\mathrm{par}}>3 / 4$.

In order to better quantify the level of agreement among the perturbative predictions, in the right-hand panels of Fig. 1 and Fig. 2, we present the comparisons of the NNLO coefficients directly. We plot the distribution of the NNLO coefficient $O C(O)$ in the top panels, while the middle and bottom panels again show the ratios of the predictions of SW and GGGH normalized to our results. The narrow gray bands in the middle and lower panels show the numerical uncertainty of our computation due to Monte Carlo integrations. We observe a very good numerical convergence of our method at NNLO. Examining these plots, we see that the agreement is generally quite good between the predictions of SW and CoLorFulNNLO and reasonably good between GGGH and CoLorFulNNLO, with the precise comparison to GGGH being hampered by the somewhat large numerical uncertainties of those predictions. We also see that significant deviations are present for small and large values of the event shapes. For example, the differences between CoLorFulNNLO and the two other predictions grow up to a factor of two for $\tau>1 / 3$. However, in this region, the contribution from the three-particle final state vanishes and the thrust distribution is 
determined by a four-jet final state. Hence, in this region, $C(\tau)$ is given by NLO corrections to four-jet production, which have been known for a long time $[21,22]$ and can also be computed by modern automated tools such as MadGraph5_aMC@NLO [23]. We find that our predictions are in complete agreement with those of MadGraph5_aMC@NLO for the thrust distribution for $\tau>1 / 3$, and with the computation of Ref. [22] for the $C$-parameter distribution for $C_{\mathrm{par}}>3 / 4$. For small values of the event shapes, we checked that our predictions are in agreement with the resummed computations obtained from SCET [24-26] expanded to $\mathcal{O}\left(\alpha_{\mathrm{s}}^{3}\right)$.

Beside the standard event shape variables discussed above, we computed for the first time predictions at NNLO accuracy for oblateness, energyenergy correlation (EEC) [7] and jet cone energy fraction (JCEF) [8]. Here, we present our results for jet cone energy fraction, which is defined as

$$
\frac{\mathrm{d} \Sigma_{\mathrm{JCEF}}}{\mathrm{d} \cos \chi}=\sum_{i} \int \mathrm{d} \sigma_{e^{+} e^{-} \rightarrow i+X} \frac{E_{i}}{Q} \delta\left(\cos \chi+\cos \theta_{i j}\right) \delta\left(\cos \chi-\frac{\vec{p}_{i} \cdot \vec{n}_{\mathrm{T}}}{\left|\vec{p}_{i}\right|}\right),
$$

where $Q$ is the center-of-mass energy, $E_{i}$ is the energy of particle $i$ (in the center-of-mass frame), $\cos \theta_{i j}$ is the cosine of the angle between the threemomenta of particles $i$ and $j$ (also in the center-of-mass frame) and $\vec{n}_{\mathrm{T}}$ is the thrust axis pointing from the heavy- to the light-jet mass hemisphere. Our physical predictions for the jet cone energy fraction at LO, NLO and NNLO accuracy together with our prediction for the NNLO coefficient $C_{\mathrm{JCEF}}(\chi)$ are presented in Fig. 3. Our code displays a good numerical convergence also for these distributions. In the left-hand panel showing the physical prediction, we have also included experimental data measured by the DELPHI Collab-
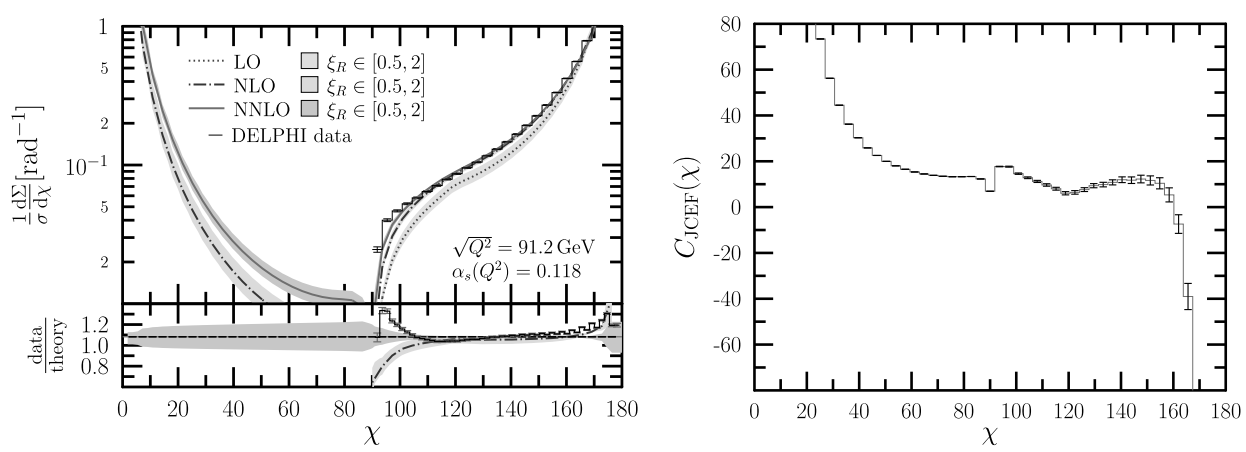

Fig. 3. Left: physical predictions for jet cone energy fraction at LO, NLO and NNLO accuracy in QCD with bands representing scale uncertainty. Data measured by the DELPHI Collaboration [27] is also shown. Right: the $C_{\mathrm{JCEF}}(\chi)$ NNLO coefficient of the jet cone energy fraction distribution. Error bars represent the numerical uncertainty coming from Monte Carlo integrations. 
oration. We observe that perturbative corrections are rather small over a wide range of angles. Hadronization corrections and detector corrections for this observable are also known to be quite small and indeed the perturbative result alone is seen to give a rather reasonable description of the data. Hence, jet cone energy fraction is a particularly simple and promising observable for the precise extraction of $\alpha_{\mathrm{s}}$ from data [27].

Finally, we turn to the computation of jet rates at NNLO accuracy. The production rate for $n$-jet events in electron-positron annihilation is given by the ratio of the $n$-jet cross section to the total hadronic cross section

$$
R_{n}\left(y_{\text {cut }}\right)=\frac{\sigma_{n}\left(y_{\text {cut }}\right)}{\sigma_{\text {tot }}}
$$

Here, the $n$-jet cross section $\sigma_{n}\left(y_{\text {cut }}\right)$ must be defined using an infraredsafe jet clustering algorithm. One class of such algorithms are the exclusive sequential recombination algorithms. Here, we focus on the Durham algorithm [29] for which the resolution variable is defined as

$$
y_{i j}=\frac{2 \min \left(E_{i}^{2}, E_{j}^{2}\right)\left(1-\cos \theta_{i j}\right)}{Q^{2}}
$$

and recombination is performed in the $E$-scheme, i.e., the four-momenta of the objects to be combined are simply added.

We present our preliminary physical predictions for $R_{3}\left(y_{\text {cut }}\right)$ in the Durham clustering algorithm in the left panel of Fig. 4 at LO, NLO and NNLO accuracy, together with measured data from the OPAL Collaboration.
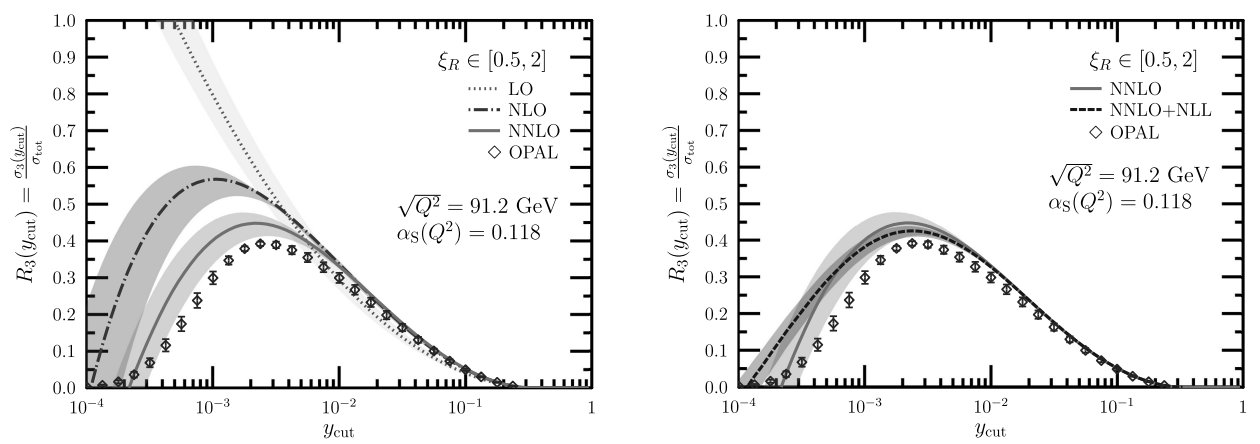

Fig. 4. Left: preliminary physical predictions for the three-jet rate $R_{3}\left(y_{\text {cut }}\right)$ in the Durham clustering algorithm at LO, NLO and NNLO accuracy in QCD with bands representing scale uncertainty. Data measured by the OPAL Collaboration [28] is also shown. Right: preliminary predictions for the three-jet rate $R_{3}\left(y_{\text {cut }}\right)$ at NNLO and NNLO+NLL accuracy. 
Although the inclusion of the NNLO corrections vastly improves the theoretical description of the measurement, a sizable difference remains between the NNLO prediction and the data for small values of the resolution parameter. We attribute this difference to missing parton shower (or resummation) and hadronization effects. In order to improve the situation, we match our perturbative prediction to the resummed result, which is known for $R_{3}\left(y_{\text {cut }}\right)$ in the two-jet limit (i.e., $y_{\text {cut }} \rightarrow 0$ ) up to next-to-leading-logarithmic (NLL) accuracy [29]. (The distribution of the two-to-three jet transition variable $y_{23}$ in the Durham algorithm is known up to NNLL accuracy [30].) This matching is performed as follows. We write the fixed order prediction for the three-jet rate at NNLO accuracy as

$$
R_{3}^{\mathrm{NNLO}}\left(y_{\text {cut }}\right)=\frac{\alpha_{\mathrm{s}}}{2 \pi} A_{3}\left(y_{\text {cut }}\right)+\left(\frac{\alpha_{\mathrm{s}}}{2 \pi}\right)^{2} B_{3}\left(y_{\text {cut }}\right)+\left(\frac{\alpha_{\mathrm{s}}}{2 \pi}\right)^{3} C_{3}\left(y_{\text {cut }}\right) .
$$

The resummed prediction at NLL accuracy can be written in the following form:

$$
R_{3}^{\mathrm{NLL}}\left(y_{\text {cut }}\right)=2\left[\Delta_{q}(Q)\right]^{2} \int_{Q_{0}}^{Q} \mathrm{~d} q \Gamma_{q}(Q, q) \Delta_{g}(q),
$$

where $Q_{0}=\sqrt{y_{\text {cut }}} Q$ and the $\Delta_{i}\left(Q^{\prime \prime}\right)$ and $\Gamma_{i}\left(Q^{\prime \prime}, Q^{\prime}\right)$ functions are given explicitly in [29] up to NLL accuracy. When evaluating Eq. (14) numerically, we use the one-loop running of $\alpha_{\mathrm{s}}$ in $\Delta_{i}\left(Q^{\prime \prime}\right)$ and $\Gamma_{i}\left(Q^{\prime \prime}, Q^{\prime}\right)$. In order to perform the matching, we expand Eq. (14) in powers of $\alpha_{\mathrm{s}}$ up to and including $\mathcal{O}\left(\alpha_{\mathrm{s}}^{3}\right)$ terms

$$
\begin{aligned}
R_{3}^{\mathrm{NLL}}\left(y_{\text {cut }}\right)= & \frac{\alpha_{\mathrm{s}}}{2 \pi} A_{3}^{\mathrm{NLL}}\left(y_{\text {cut }}\right)+\left(\frac{\alpha_{\mathrm{s}}}{2 \pi}\right)^{2} B_{3}^{\mathrm{NLL}}\left(y_{\text {cut }}\right)+\left(\frac{\alpha_{\mathrm{s}}}{2 \pi}\right)^{3} C_{3}^{\mathrm{NLL}}\left(y_{\text {cut }}\right) \\
& +\mathcal{O}\left(\alpha_{\mathrm{s}}^{4}\right) .
\end{aligned}
$$

Our final expression at NNLO+NLL accuracy is then given by

$$
\begin{aligned}
R_{3}^{\mathrm{NNLO}+\mathrm{NLL}}\left(y_{\text {cut }}\right)= & R_{3}^{\mathrm{NLL}}\left(y_{\text {cut }}\right)+\frac{\alpha_{\mathrm{s}}}{2 \pi}\left[A_{3}\left(y_{\text {cut }}\right)-A_{3}^{\mathrm{NLL}}\left(y_{\text {cut }}\right)\right] \\
& +\left(\frac{\alpha_{\mathrm{s}}}{2 \pi}\right)^{2}\left[B_{3}\left(y_{\text {cut }}\right)-B_{3}^{\mathrm{NLL}}\left(y_{\text {cut }}\right)\right] \\
& +\left(\frac{\alpha_{\mathrm{s}}}{2 \pi}\right)^{3}\left[C_{3}\left(y_{\text {cut }}\right)-C_{3}^{\mathrm{NLL}}\left(y_{\text {cut }}\right)\right] .
\end{aligned}
$$

The right-hand panel of Fig. 4 shows the preliminary results of this matching procedure. We indeed see a marked improvement of the theoretical description, together with a significant reduction in the relative scale uncertainty below $y_{\text {cut }} \sim 10^{-2}$. Since jet rates computed using different jet algorithms can have different sensitivities to non-perturbative effects, it would be interesting to extend these results to other jet clustering algorithms as well. 


\section{Conclusions}

In this contribution, we briefly outlined the CoLorFulNNLO subtraction method for computing NNLO QCD corrections for processes with colorless initial states. As a first application, the method was used to compute physical observables in three-jet production in electron-positron annihilation. After validating our numerical program by comparisons to existing computations, we presented NNLO QCD results for jet cone energy fraction, which has not been computed at NNLO accuracy before. We find that the perturbative corrections for this observable are rather small for a wide range of angles. This, together with the smallness of the hadronization and detector corrections, makes the jet cone energy fraction a very promising observable for the precise extraction of the strong coupling from data. Finally, we presented preliminary results for the three-jet rate in the Durham algorithm at NNLO and NNLO + NLL accuracy.

\section{REFERENCES}

[1] Z. Bern, L.J. Dixon, D.A. Kosower, S. Weinzierl, Nucl. Phys. B 489, 3 (1997) [arXiv:hep-ph/9610370].

[2] Z. Bern, L.J. Dixon, D.A. Kosower, Nucl. Phys. B 513, 3 (1998) [arXiv:hep-ph/9708239].

[3] L.W. Garland et al., Nucl. Phys. B 627, 107 (2002) [arXiv: hep-ph/0112081].

[4] L.W. Garland et al., Nucl. Phys. B 642, 227 (2002) [arXiv:hep-ph/0206067].

[5] A. Gehrmann-De Ridder, T. Gehrmann, E.W.N. Glover, G. Heinrich, J. High Energy Phys. 0712, 094 (2007) [arXiv:0711.4711 [hep-ph]].

[6] S. Weinzierl, J. High Energy Phys. 0906, 041 (2009) [arXiv:0904.1077 [hep-ph]].

[7] V. Del Duca et al., Phys. Rev. Lett. 117, 152004 (2016) [arXiv: 1603.08927 [hep-ph]].

[8] V. Del Duca et al., Phys. Rev. D 94, 074019 (2016) [arXiv:1606.03453 [hep-ph]].

[9] A. Gehrmann-De Ridder, T. Gehrmann, E.W.N. Glover, G. Heinrich, Phys. Rev. Lett. 100, 172001 (2008) [arXiv:0802.0813 [hep-ph]].

[10] S. Weinzierl, Eur. Phys. J. C 71, 1565 (2011) [Erratum ibid. 71, 1717 (2011)] [arXiv: 1011.6247 [hep-ph]].

[11] S. Frixione, Z. Kunszt, A. Signer, Nucl. Phys. B 467, 399 (1996) [arXiv:hep-ph/9512328].

[12] S. Catani, M.H. Seymour, Nucl. Phys. B 485, 291 (1997) [Erratum ibid. 510, 503 (1998)] [arXiv:hep-ph/9605323]. 
[13] Z. Nagy, Z. Trócsányi, Nucl. Phys. B 486, 189 (1997) [arXiv: hep-ph/9610498].

[14] G. Somogyi, Z. Trócsányi, Acta Phys. Chym. Debr. XL, 101 (2006) [arXiv:hep-ph/0609041].

[15] G. Somogyi, J. High Energy Phys. 0905, 016 (2009) [arXiv:0903.1218 [hep-ph]].

[16] G. Somogyi, Z. Trócsányi, V. Del Duca, J. High Energy Phys. 0701, 070 (2007) [arXiv:hep-ph/0609042].

[17] G. Somogyi, Z. Trócsányi, J. High Energy Phys. 0701, 052 (2007) [arXiv: hep-ph/0609043].

[18] G. Somogyi, Z. Trócsányi, J. High Energy Phys. 0808, 042 (2008) [arXiv:0807.0509 [hep-ph]]; U. Aglietti et al., J. High Energy Phys. 0809, 107 (2008) [arXiv:0807.0514 [hep-ph]]; P. Bolzoni, S.O. Moch, G. Somogyi, Z. Trócsányi, J. High Energy Phys. 0908, 079 (2009) [arXiv:0905.4390 [hep-ph]]; P. Bolzoni, G. Somogyi, Z. Trócsányi, J. High Energy Phys. 1101, 059 (2011) [arXiv:1011.1909 [ hep-ph]]; V. Del Duca, G. Somogyi, Z. Trócsányi, J. High Energy Phys. 1306, 079 (2013) [arXiv: 1301.3504 [hep-ph]]; G. Somogyi, J. High Energy Phys. 1304, 010 (2013) [arXiv: 1301.3919 [hep-ph]].

[19] A. Gehrmann-De Ridder, T. Gehrmann, E.W.N. Glover, G. Heinrich, Comput. Phys. Commun. 185, 3331 (2014) [arXiv:1402.4140 [hep-ph]].

[20] A. Heister et al. [ALEPH Collaboration], Eur. Phys. J. C 35, 457 (2004).

[21] A. Signer, L.J. Dixon, Phys. Rev. Lett. 78, 811 (1997) [arXiv:hep-ph/9609460].

[22] Z. Nagy, Z. Trócsányi, Phys. Rev. Lett. 79, 3604 (1997) [arXiv: hep-ph/9707309].

[23] J. Alwall et al., J. High Energy Phys. 1407, 079 (2014) [arXiv: 1405.0301 [hep-ph]].

[24] T. Becher, M.D. Schwartz, J. High Energy Phys. 0807, 034 (2008) [arXiv:0803.0342 [hep-ph]].

[25] Y.T. Chien, M.D. Schwartz, J. High Energy Phys. 1008, 058 (2010) [arXiv: 1005.1644 [hep-ph]].

[26] A.H. Hoang, D.W. Kolodrubetz, V. Mateu, I.W. Stewart, Phys. Rev. D 91, 094017 (2015) [arXiv: 1411.6633 [hep-ph]].

[27] P. Abreu et al. [DELPHI Collaboration], Eur. Phys. J. C 14, 557 (2000) [arXiv: hep-ex/0002026].

[28] P. Pfeifenschneider et al. [JADE and OPAL collaborations], Eur. Phys. J. C 17, 19 (2000) [arXiv:hep-ex/0001055].

[29] S. Catani et al., Phys. Lett. B 269, 432 (1991).

[30] A. Banfi, H. McAslan, P.F. Monni, G. Zanderighi, Phys. Rev. Lett. 117, 172001 (2016) [arXiv:1607.03111 [hep-ph]]. 